\title{
Prevalence and Possible Role of Candida Species in Patients with Psoriasis: A Systematic Review and Meta-Analysis
}

\author{
Aldona Pietrzak (D), ${ }^{1}$ Ewelina Grywalska $\mathbb{D}^{2},{ }^{2}$ Mateusz Socha $\mathbb{D}^{3},{ }^{3}$ Jacek Roliński $\left(\mathbb{D},{ }^{2}\right.$ \\ Kinga Franciszkiewicz-Pietrzak, ${ }^{4}$ Lidia Rudnicka, ${ }^{5}$ Marcin Rudzki, ${ }^{6}$ and Dorota Krasowska ${ }^{1}$ \\ ${ }^{1}$ Department of Dermatology, Venereology and Pediatric Dermatology, Medical University of Lublin, Ul. Radziwillowska 13, \\ 20-080 Lublin, Poland \\ ${ }^{2}$ Department of Clinical Immunology and Immunotherapy, Medical University of Lublin, Ul. Chodźki 4a, 20-093 Lublin, Poland \\ ${ }^{3}$ Department of Internal Medicine and Cardiology, First Military Clinical Hospital with the Outpatient Clinic, Al. Ractawickie 23, \\ 20-048 Lublin, Poland \\ ${ }^{4}$ Department of Surgical Oncology, Medical University of Lublin, Ul. Staszica 11, 20-081 Lublin, Poland \\ ${ }^{5}$ Department of Dermatology, Medical University of Warsaw, Ul. Koszykowa 82a, 02-008 Warsaw, Poland \\ ${ }^{6}$ Chair and Department of Jaw Orthopaedics, Medical University of Lublin, Ul. Karmelicka 7, 20-081 Lublin, Poland
}

Correspondence should be addressed to Aldona Pietrzak; aldonkapietrzak@o2.pl

Received 12 January 2018; Accepted 8 April 2018; Published 6 May 2018

Academic Editor: Maria Rosaria Catania

Copyright ( 2018 Aldona Pietrzak et al. This is an open access article distributed under the Creative Commons Attribution License, which permits unrestricted use, distribution, and reproduction in any medium, provided the original work is properly cited.

\begin{abstract}
Although fungal colonization is implicated in the pathogenesis of psoriasis, its prevalence remains unclear. The aim of this systematic review and meta-analysis was to provide an overview on the prevalence of Candida species in patients with psoriasis. We searched databases (MEDLINE, EMBASE, Cochrane Central Register of Controlled Trials, and http://clinicaltrials.gov) to identify studies involving subjects of any age with an established diagnosis of psoriasis and healthy controls, who were tested for carriage of Candida spp. on the skin or mucosal membranes (or saliva and stool), or presented with clinical candidiasis with microbiologically confirmed etiology. We identified nine cross-sectional studies including a total of 1038 subjects with psoriasis (psoriatics) and 669 controls. We found Candida species detection rates for psoriatics were significantly higher than those in the controls, especially in the oral mucosa milieux. These results suggest psoriasis may be one of the systemic diseases that predispose to oral Candida spp. carriage and infection.
\end{abstract}

\section{Introduction}

Psoriasis is a common chronic inflammatory disease of the skin and joint, affecting approximately $2-4 \%$ of the general population [1]. Depending on the study population, the prevalence of psoriasis varies from $0.09 \%$ in the United Republic of Tanzania [2] to $11.4 \%$ in Norway [3].

Early concepts of the etiology of psoriasis focused primarily on keratinocyte hyperproliferation. However, dysregulation of the immune system is now recognized as a critical event in the pathogenesis of psoriasis. The inflammatory cascade of psoriasis involves complex interactions between keratinocytes, dendritic cells, neutrophils, and particularly
T cells [4]. Psoriasis is now considered to be an organspecific $\mathrm{T}$ cell-driven inflammatory disease, with interplay among Th1, Th9, Th17, Treg, and Th22 cells contributing to the development of the disease, although the nature of the antigen (autoantigen or microbial) that activates psoriatic $\mathrm{T}$ cells remains controversial $[5,6]$.

Over the past several years, the association between the microbiome and inflammatory skin diseases has been increasingly recognized [7]. It is hypothesized that microbial dysbiosis of the skin and mucosa could trigger an exaggerated immune response in a susceptible host, inducing a persistent inflammatory process associated with autoimmune disorders [8]. Several microorganisms (including bacteria, viruses, and 
fungi) were found to act as superantigens that activate specific $\mathrm{T}$ cells and initiate, exacerbate, and maintain chronic inflammation in skin disease [9]. This interconnection has been most thoroughly studied for Staphylococcus aureus skin colonization in atopic dermatitis and psoriasis [10, 11].

In addition to bacteria, fungi have also been implicated in stimulating skin-associated lymphoid tissue. The Candida species are a part of the normal human microbiota, frequently colonizing the skin and mucosal membranes of gastrointestinal and genitourinary tracts [12]. These common commensal organisms are capable of causing opportunistic infection following the disruption of the normal microbiome, a breach in the integrity of the mucocutaneous barrier, or immunodeficiency [13].

Although dysbiosis and the role of the microbiome in the pathogenesis of inflammatory skin diseases have been intensively investigated, fungal colonization or infection has received minimal attention. Therefore, the aim of our systematic review and meta-analysis was to provide an overview on the prevalence and colonization with Candida spp. in patients with psoriasis.

\section{Methods}

This meta-analysis was conducted in accordance with the Preferred Reporting Items for Systematic Reviews and Meta-Analyses (PRISMA) statement [14].

2.1. Search Strategy. Two researchers (Mateusz Socha and Kinga Franciszkiewicz-Pietrzak) performed independent, comprehensive searches of the following databases, from inception through May 20, 2017: MEDLINE (accessed by PubMed), EMBASE, Cochrane Central Register of Controlled Trials, and http://clinicaltrials.gov. The following search terms were used: "psoriasis," "psoriatic," "psoriatics," "Candida," "Candida spp.," "Candida albicans," "candidiasis," "fungal infection," "carrier," "carriage," "mycosis," "oral mucosa," "tongue mucosa," "gut mucosa," "saliva," "stool," "skin," "dermal," "swab," "epidemiological study," and "cross-sectional study" and combinations thereof. The search was limited to human studies but not restricted to any particular language or publication date. Reference lists from all available review articles were also searched manually. Database search by keywords gave 128 results; additionally, 6 records were found manually by reference lists. From the total number of 134 records, 7 occurred to be duplicates. Among 118 excluded articles in 76, the detection rate of Candida spp. was not studied, 30 were review articles, and 12 were case reports. Authors concluded that 9 articles were eligible for data extraction.

2.2. Study Selection. Published studies were considered eligible for the meta-analysis whenever they included subjects of any age with an established diagnosis of psoriasis and healthy controls, who were tested for carriage of Candida spp. on the skin or mucosal membranes (or saliva and stool), or presented with clinical candidiasis with microbiologically confirmed etiology.

To avoid double counting of patients included in more than one article by the same authors or research groups, patient recruitment periods were evaluated. Titles and abstracts of retrieved articles were independently evaluated by two researchers (Mateusz Socha and Kinga Franciszkiewicz-Pietrzak). Full-text articles were reviewed when abstracts did not provide sufficient information about inclusion and exclusion criteria. Full text of each article was reviewed independently, and its eligibility for inclusion in the meta-analysis was evaluated. Results were compared, and discrepancies were resolved through discussion and, if necessary, the inclusion of the third researcher (Ewelina Grywalska). The whole process is illustrated in Figure 1.

2.3. Primary Outcomes. The primary outcome of interest was a dichotomous variable: Candida spp. detection rate in psoriasis patients and healthy controls.

2.4. Data Extraction. Extracted data included information about the study design, characteristics and number of participants, source of examined material (skin versus mucosa), and Candida spp. detection rates. The details were summarized in standard extraction sheets, independently by two authors (Ewelina Grywalska and Jacek Roliński).

2.5. Statistical Analysis. Meta-analysis was conducted with Statistica 10 package (StatSoft, Tulsa, OK, USA), using the random effects model. The outcomes were calculated as odds ratios (OR) with their 95\% confidence intervals (95\% CIs). Homogeneity of the studies was verified with T2-test based on the weighted least square method. Post hoc sensitivity analysis was conducted to examine whether the overall finding was robust to an outlying study. In sensitivity analysis, sequential meta-analyses were repeated in which each study was excluded. Subgroup analyses were also carried out to determine Candida spp. detection rates from the skin and mucosa and/or in children and adults. The results of all tests were considered significant at $p<0.05$.

\section{Results}

Of 134 records initially identified, titles and abstracts of 127 studies were assessed for eligibility after the exclusion of duplicates (Figure 1). A total of 118 records were excluded because the studies did not analyze Candida spp. detection rates $(n=76)$, or the retrieved publications were review articles $(n=30)$ or case reports $(n=12)$. Eventually, nine cross-sectional studies including a total of 1038 psoriatics and 669 controls [15-23] were available for analysis.

3.1. Description of Included Studies. Six analyzed studies included only adults [17-21, 23], and two studies involved both adults and children $[16,22]$. Authors of one study did not specify if their subjects were older than 18 years [15]. Six studies solely analyzed material from mucosal membranes $[15,16,19-21,23]$, one solely analyzed material from the skin [17], and two included both mucosal and skin swabs $[18,22]$. Two studies were conducted in Israel $[16,18]$, two in Jordan $[19,20]$, and the remaining in Germany [15], Sweden [17], Brazil [21] Iran [22], and Thailand [23]. All studies included patients with established diagnosis of psoriasis, both women and men. Detailed characteristics of all included studies are presented in Table 1. 


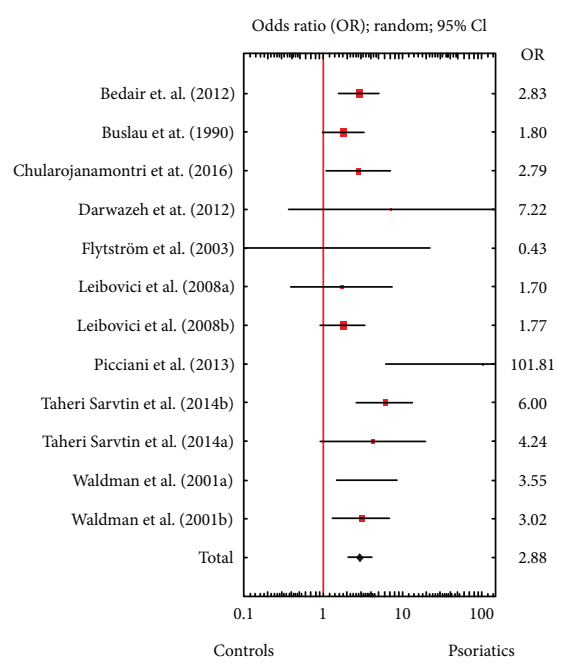

(a)

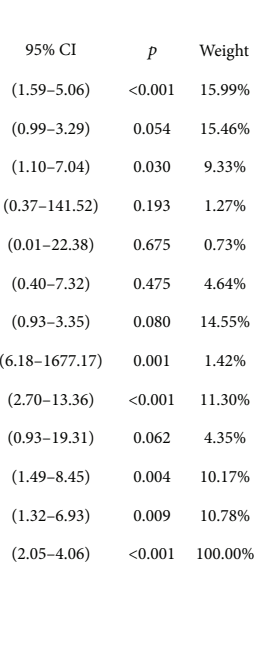

$(1.59-5.06) \quad<0.001 \quad 15.99 \%$

$(0.99-3.29) \quad 0.054 \quad 15.46 \%$

$\begin{array}{lll}(1.10-7.04) & 0.030 \quad 9.33 \%\end{array}$

$\begin{array}{lll}(0.37-141.52) & 0.193 & 1.27 \%\end{array}$

$\begin{array}{lll}0.01-22.38) & 0.675 & 0.73 \%\end{array}$

$0.475 \quad 4.64 \%$

$.080 \quad 14.55 \%$

$1.42 \%$

$001 \quad 11.30 \%$

$10.17 \%$

$10.78 \%$

$100.00 \%$

FIgURe 1: (a) Meta-analysis of nine studies (among them, three contained two different types of examined material) comparing odds ratios (ORs) for the detection of Candida spp. from the skin or mucosal membranes of psoriasis patients and healthy controls of any age. (b) Metaanalysis of six studies (among them, one contained two different types of examined material) comparing odds ratios (ORs) for the detection of Candida spp. from the skin or mucosal membranes of adult psoriasis patients and healthy controls.

TABLE 1: Characteristics of studies included in the meta-analysis.

\begin{tabular}{|c|c|c|c|c|c|c|}
\hline \multirow{2}{*}{ Authors } & \multirow{2}{*}{ Material } & \multirow{2}{*}{ Age group } & \multicolumn{2}{|c|}{ Study subjects $(n)$} & \multicolumn{2}{|c|}{ Positive subjects $(n(\%))$} \\
\hline & & & Patients & Controls & Patients & Controls \\
\hline Buslau et al. (1990) & Stool & Unspecified & 343 & 50 & $233(68 \%)$ & $27(54 \%)$ \\
\hline Waldman et al. (2001a) & Saliva & Children and adults & 50 & 50 & $39(78 \%)$ & $25(50 \%)$ \\
\hline Waldman et al. (2001b) & Stool & Children and adults & 50 & 50 & $36(72 \%)$ & $23(46 \%)$ \\
\hline Flytström et al. (2003) & Axilla and groin & Adults & 45 & 19 & $0(0 \%)$ & $0(0 \%)$ \\
\hline Leibovici et al. (2008a) & Axilla and groin & Adults & 100 & 100 & $5(5 \%)$ & $3(3 \%)$ \\
\hline Leibovici et al. (2008b) & Tongue & Adults & 100 & 100 & $32(32 \%)$ & $21(21 \%)$ \\
\hline Bedair et al. (2012) & Normal oral mucosa & Adults & 100 & 100 & $69(69 \%)$ & $44(44 \%)$ \\
\hline Darwazeh et al. (2012) & Oral mucosal lesions & Adults & 100 & 100 & $3(3 \%)$ & $0(0 \%)$ \\
\hline Picciani et al. (2013) & Normal oral mucosa & Adults & 140 & 140 & $37(26 \%)$ & $0(0 \%)$ \\
\hline Taheri Sarvtin et al. (2014a) & Normal skin & Children and adults & 100 & 50 & $15(15 \%)$ & $2(4 \%)$ \\
\hline Taheri Sarvtin et al. (2014b) & Normal oral mucosa & Children and adults & 100 & 50 & $60(60 \%)$ & $10(20 \%)$ \\
\hline Chularojanamontri et al. (2016) & Normal oral mucosa & Adults & 60 & 60 & $18(30 \%)$ & $8(13.3 \%)$ \\
\hline
\end{tabular}

3.2. Effect of Intervention. A random effects model pooling the results demonstrated that Candida spp. detection rates for psoriatics were significantly higher than those in the controls. The OR for isolation of Candida spp. from either the skin or the mucosal membranes of patients with psoriasis was 2.88 (95\% CI: 2.05-4.06, $p<0.001$ ), when both children and adults were included (Figure 1(a)), and 2.65 (95\% CI: $1.51-4.65, p=0.001$ ) for solely adults (Figure 1(b)). Statistically significantly higher Candida spp. detection rates were also documented for mucosal membranes, either in all studies $(\mathrm{OR}=3.00,95 \% \mathrm{CI}$ : 2.03-4.43, $p<0.001$; Figure $2(\mathrm{a}))$ or solely in studies involving adults $(\mathrm{OR}=3.05,95 \% \mathrm{CI}: 1.55-$ 5.99, $p=0.001$; Figure 2(b)). However, psoriasis patients and controls did not differ significantly in terms of Candida spp. isolation rates from the skin (Figures $3(\mathrm{a})$ and $3(\mathrm{~b})$ ).

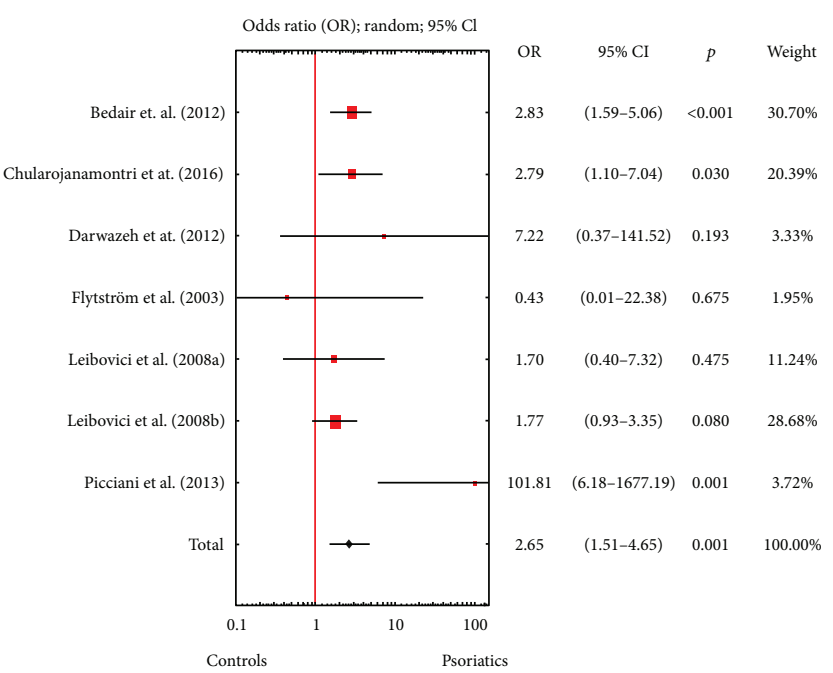

(b) 


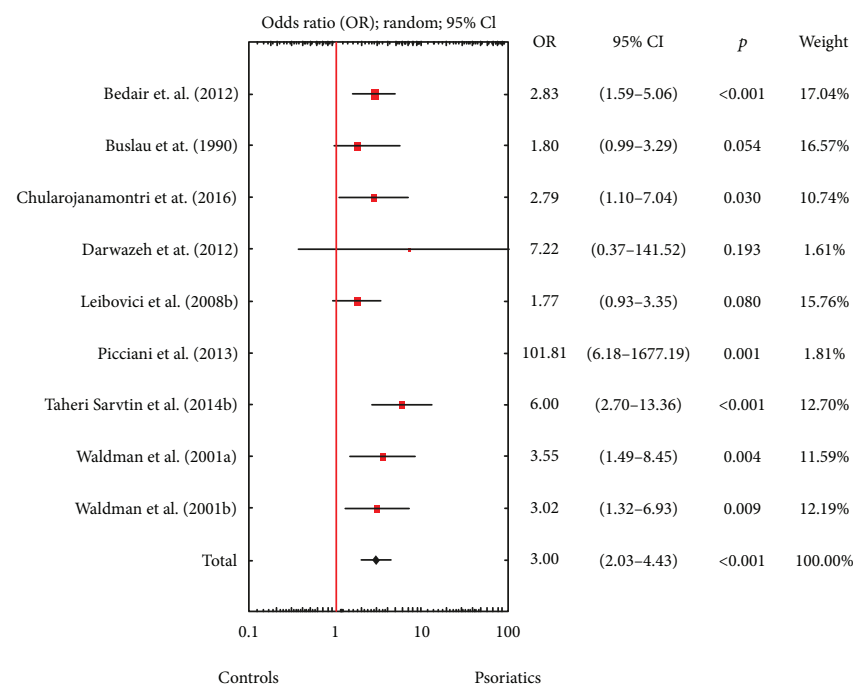

(a)

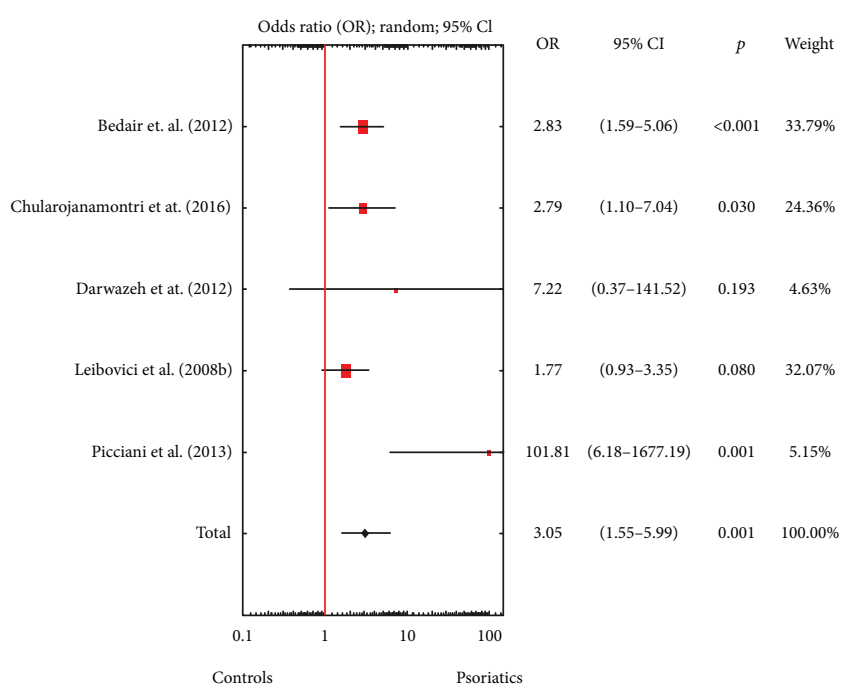

(b)

Figure 2: (a) Meta-analysis of eight studies (among them, one contained two different types of examined material) comparing odds ratios (ORs) for the detection of Candida spp. from mucosal membranes of psoriasis patients and healthy controls of any age. (b) Meta-analysis of five studies comparing odds ratios (ORs) for the detection of Candida spp. from mucosal membranes of adult psoriasis patients and healthy controls.

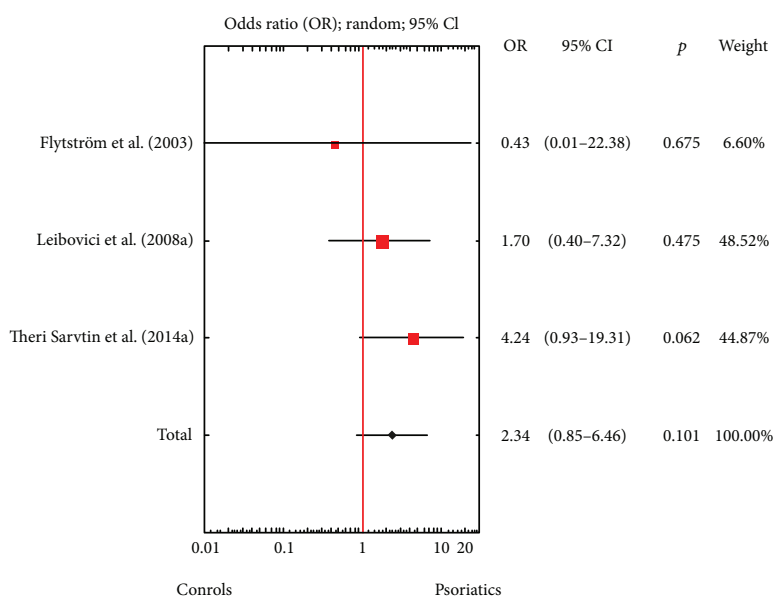

(a)

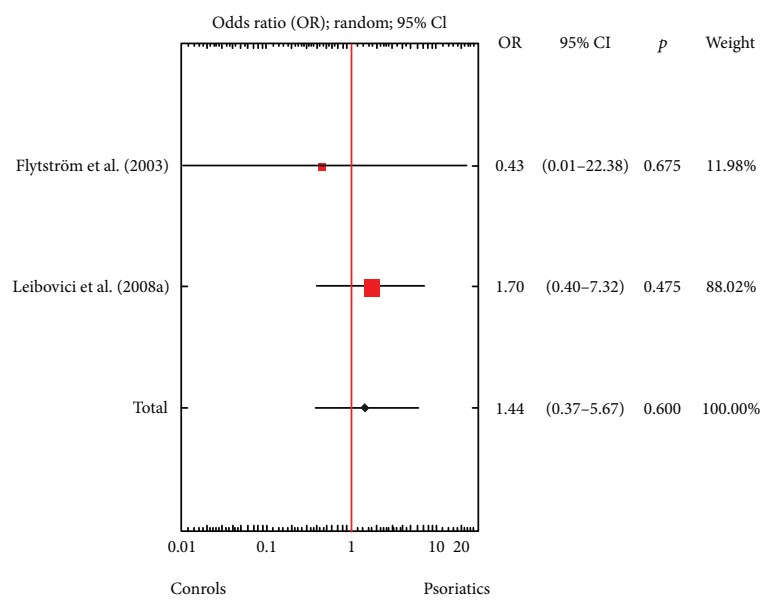

(b)

FIGURE 3: (a) Meta-analysis of three studies comparing odds ratios (ORs) for the detection of Candida spp. from the skin of psoriasis patients and healthy controls of any age. (b) Meta-analysis of two studies comparing odds ratios (ORs) for the detection of Candida spp. from the skin of adult psoriasis patients and healthy controls.

The underlying mechanism for the association between psoriasis and Candida spp. has not yet been clearly identified. In healthy adults and children, the yeast-like fungi Candida spp. are not pathogenic and they are a commensal of the normal microbiome of the skin, oral cavity, gastrointestinal tract, and vaginal mucosa [13]. However, the abundance of Candida spp. on the skin may increase with altered immune function. Similarly to psoriasis, a higher prevalence of Candida colonization has been found in other inflammatory skin disorders, including atopic dermatitis [24].

Yeast-like fungi Candida play an important role in triggering psoriasis flares. Candida spp. antigens, especially Candida albicans surface proteins have been shown to have superantigen-like effects, resulting in the activation of $\mathrm{T}$ lymphocytes independently of antigen presentation and excessive release of proinflammatory cytokines $[25,26]$. These cytokines, especially interleukin-23 (IL-23), promote the proliferation and survival of Th17 cells, which are essential for host defense against C. albicans [12]. In turn, Th17 cells release IL-17, which recruits neutrophils and contributes to Candida spp. clearance through releasing high amounts of antimicrobial peptides (AMPs), direct phagocytosis, and formation of neutrophil extracellular traps [27]. Finally, besides Th17 cells, skin resident and recruited Th9 cells bridge the innate and adaptive immune response against C. albicans infection [28]. 
TABLE 2: Post hoc sensitivity analysis for studies included in the meta-analysis (each study excluded).

\begin{tabular}{|c|c|c|c|c|c|}
\hline Excluded study & OR & SE & $95 \% \mathrm{CI}$ & $p$ & Weight \\
\hline Bedair et al. (2012) & 2.94 & 0.61 & $1.96-4.43$ & $<0.001$ & $84.01 \%$ \\
\hline Buslau et al. (1990) & 3.14 & 0.59 & $2.17-4.53$ & $<0.001$ & $84.54 \%$ \\
\hline Chularojanamontri et al. (2016) & 2.93 & 0.57 & $2.00-4.29$ & $<0.001$ & $90.67 \%$ \\
\hline Darwazeh et al. (2012) & 2.86 & 0.52 & $2.01-4.07$ & $<0.001$ & $98.73 \%$ \\
\hline Flytström et al. (2003) & 2.93 & 0.52 & $2.07-4.14$ & $<0.001$ & $99.27 \%$ \\
\hline Leibovici et al. (2008a) & 2.98 & 0.55 & $2.07-4.28$ & $<0.001$ & $95.36 \%$ \\
\hline Leibovici et al. (2008b) & 3.13 & 0.59 & $2.17-4.51$ & $<0.001$ & $85.45 \%$ \\
\hline Picciani et al. (2013) & 2.66 & 0.35 & $2.05-3.45$ & $<0.001$ & $98.58 \%$ \\
\hline Taheri Sarvtin et al. (2014a) & 2.85 & 0.53 & $1.98-4.09$ & $<0.001$ & $95.65 \%$ \\
\hline Taheri Sarvtin et al. (2014b) & 2.57 & 0.42 & $1.87-3.53$ & $<0.001$ & $88.70 \%$ \\
\hline Waldman et al. (2001a) & 2.84 & 0.55 & $1.94-4.15$ & $<0.001$ & $89.83 \%$ \\
\hline Waldman et al. (2001b) & 2.90 & 0.57 & $1.97-4.27$ & $<0.001$ & $89.22 \%$ \\
\hline Overall effect & 2.88 & 0.50 & $2.05-4.06$ & $<0.001$ & $100.00 \%$ \\
\hline
\end{tabular}

The mechanisms involved in host defense against C. albicans share similar pathways associated with the pathogenesis of psoriasis. For example, it was recently shown that a Th9 cell subset, which secretes large quantities of IL-9, is increased in psoriatic skin lesions [6]. In addition, the IL23/Th17/IL-17 pathway is one of the most important inflammatory processes in psoriasis, and its therapeutic blockade with biological agents is highly effective in the treatment of moderate to severe psoriasis [29]. However, at the same time, these pharmacological interventions affect antifungal immune responses and may partially promote increased prevalence of commensal colonization as well as the invasive growth of $C$. albicans $[30,31]$.

Subgroup analysis of our meta-analysis revealed significantly higher detection rates for Candida spp. on mucosal membranes. Recently available culture-independent methods of microorganisms profiling have improved our understanding of the microbiome and its impact on health [7]. So far, research has focused mainly on the skin microbiome, while data on the association between the gut microbiota and the skin disease is limited [32]. It is becoming increasingly apparent that gut microbiota might be able not only to regulate the local gastrointestinal immune system but also to affect the systemic immune system and thereby in turn influence other organs such as the skin [33]. From the evolutionary point of view, this interplay between the environmental microflora and the immune system at the barriers of the body is essential for educating the immune system and thus for our survival. However, in some genetically predisposed individuals, bacteria or fungi on the mucous membranes may lead to the activation of the local innate immune system and, in turn, induce an adaptive immune response [34]. These recent findings partially explain the presence of gastrointestinal symptoms in patients with psoriasis as well as coexistence with inflammatory bowel diseases $[35,36]$.

All analyzed studies in our meta-analysis indicated a higher prevalence of Candida spp. colonization in the oral cavity of psoriatic patients. These results suggest that psoriasis can be one of the systemic diseases that predispose to oral Candida spp. carriage and infection. Nevertheless, there are conflicting results concerning the association between oral candidiasis and systemic antipsoriatic treatment. In a study by Chularojanamontri et al. [23], the presence of oral Candida spp. infection assessed by culturing oral swabs was significantly higher in patients with psoriasis receiving immunosuppressive therapy. On the other hand, Bedair et al. [19] and Picciani et al. [21] showed a lack of this relationship. The methods used to confirm Candida spp. presence were concentrated oral rinse test and cytopathological examination, respectively. Discrepancies between studies may stem from difficulties in the differential diagnosis of oral psoriasis due to overlapping clinical and histological features with candidiasis. Candidiasis may clinically resemble psoriatic erythematous patches of the oral mucosa, and the two conditions share some similar features on histopathological examination such as hyperplastic rete ridges and intraepithelial neutrophils [37, 38]. Negative results of culture PAS stain of biopsy material or failure of antifungal treatment may help exclude Candida spp. etiology. In the view of the current results, further studies are needed to clarify the main factors predisposing patients with psoriasis for increased oral Candida spp. colonization and developing an active infection.

Surprisingly, in our study, psoriasis patients and controls did not differ significantly in the rate of Candida spp. isolated from the skin. There are two possible explanations of this observation. First, the results are based on the limited number of studies with considerable heterogeneity among them. Second, C. albicans growth could be inhibited by AMPs, which are excessively produced in a lesional psoriatic skin [39]. AMPs, also known as host defense proteins, are key molecules in the cutaneous innate immune system. They exhibit broad-spectrum killing activity against bacteria, fungi, and several parasites [40]. Calprotectin, a heterocomplex of the two calcium-binding proteins S100A8 and S100A9, is one of the most common skin-derived AMPs [41]. Calprotectin exerts fungistatic activity toward C. albicans [42]. It was also shown that calprotectin expression in the epidermis is upregulated in inflammatory skin diseases, such as psoriasis [43]. 
In conclusion, increased Candida colonization has been confirmed in subjects with psoriasis. This result is of great clinical importance due to the potential risk for Candida infections during treatment with novel biologic drugs such as IL-17 inhibitors, which significantly affect the antifungal immune response. Future studies are needed to investigate the interaction between Candida spp. colonization and immune system alterations in order to obtain possible new microbiome-targeted therapeutic options.

\section{Conflicts of Interest}

The authors declare that they have no conflicts of interest.

\section{Acknowledgments}

The authors would like to express their gratitude to Proper Medical Writing Ltd. for their assistance in writing this manuscript and to Dr. Szymon Bruzewicz (SciencePro) for the meta-analysis. The study was supported by the Medical University of Lublin funds (Grant no. DS 168/ 2017 and DS 460/2017).

\section{References}

[1] W. H. Boehncke and M. P. Schön, "Psoriasis," The Lancet, vol. 386, no. 9997, pp. 983-994, 2015.

[2] S. Gibbs, "Skin disease and socioeconomic conditions in rural Africa: Tanzania," International Journal of Dermatology, vol. 35, no. 9, pp. 633-639, 1996.

[3] K. Danielsen, A. O. Olsen, T. Wilsgaard, and A. S. Furberg, "Is the prevalence of psoriasis increasing? A 30-year follow-up of a population-based cohort," British Journal of Dermatology, vol. 168, no. 6, pp. 1303-1310, 2013.

[4] F. C. Eberle, J. Brück, J. Holstein, K. Hirahara, and K. Ghoreschi, "Recent advances in understanding psoriasis," F1000Research, vol. 5, 2016.

[5] Y. Cai, C. Fleming, and J. Yan, "New insights of T cells in the pathogenesis of psoriasis," Cellular \& Molecular Immunology, vol. 9, no. 4, pp. 302-309, 2012.

[6] C. Schlapbach, A. Gehad, C. Yang et al., "Human TH9 cells are skin-tropic and have autocrine and paracrine proinflammatory capacity," Science Translational Medicine, vol. 6, no. 219, article 219ra8, 2014.

[7] B. Dréno, E. Araviiskaia, E. Berardesca et al., "Microbiome in healthy skin update for dermatologists," Journal of the European Academy of Dermatology and Venereology, vol. 30, no. 12, pp. 2038-2047, 2016.

[8] M. Maguire and G. Maguire, "The role of microbiota and probiotics and prebiotics in skin health," Archives of Dermatological Research, vol. 309, no. 6, pp. 411-421, 2017.

[9] E. S. Macias, F. A. Pereira, W. Rietkerk, and B. Safai, "Superantigens in dermatology," Journal of the American Academy of Dermatology, vol. 64, no. 3, pp. 455-472, 2011.

[10] J. E. E. Totté, W. T. van der Feltz, M. Hennekam, A. van Belkum, E. J. van Zuuren, and S. G. M. A. Pasmans, "Prevalence and odds of Staphylococcus aureus carriage in atopic dermatitis: a systematic review and meta-analysis," British Journal of Dermatology, vol. 175, no. 4, pp. 687-695, 2016.

[11] J. E. E. Totté, W. T. van der Feltz, L. G. M. Bode, A. van Belkum, E. J. van Zuuren, and S. G. M. A. Pasmans, "A systematic review and meta-analysis on Staphylococcus aureus carriage in psoriasis acne and rosacea," European Journal of Clinical Microbiology \& Infectious Diseases, vol. 35, no. 7, pp. 1069-1077, 2016.

[12] N. Hernández-Santos and S. L. Gaffen, "Th17 cells in immunity to Candida albicans," Cell Host \& Microbe, vol. 11, no. 5, pp. 425-435, 2012.

[13] A. Kühbacher, A. Burger-Kentischer, and S. Rupp, "Interaction of Candida species with the skin," Microorganisms, vol. 5, no. 2, 2017.

[14] D. Moher, A. Liberati, J. Tetzlaff, D. G. Altman, and for the PRISMA group, "Preferred reporting items for systematic reviews and meta-analyses: the PRISMA statement," $B M J$, vol. 339, no. jul21 1, p. b2535, 2009.

[15] M. Buslau, I. Menzel, and H. Holzmann, "Fungal flora of human faeces in psoriasis and atopic dermatitis," Mycoses, vol. 33, no. 2, pp. 90-94, 1990.

[16] A. Waldman, A. Gilhar, L. Duek, and I. Berdicevsky, "Incidence of Candida in psoriasis-a study on the fungal flora of psoriatic patients," Mycoses, vol. 44, no. 3-4, pp. 77-81, 2001.

[17] I. Flytström, I. M. Bergbrant, J. Bråred, and L. L. Brandberg, "Microorganisms in intertriginous psoriasis: no evidence of Candida," Acta Dermato-Venereologica, vol. 83, no. 2, pp. 121-123, 2003.

[18] V. Leibovici, R. Alkalay, K. Hershko et al., "Prevalence of Candida on the tongue and intertriginous areas of psoriatic and atopic dermatitis patients," Mycoses, vol. 51, no. 1, pp. 63-66, 2008.

[19] A. A. Bedair, A. M. G. Darwazeh, and M. M. Al-Aboosi, "Oral candida colonization and candidiasis in patients with psoriasis," Oral Surgery, Oral Medicine, Oral Pathology and Oral Radiology, vol. 114, no. 5, pp. 610-615, 2012.

[20] A. M. Darwazeh, M. M. Al-Aboosi, and A. A. Bedair, "Prevalence of oral mucosal lesions in psoriatic patients: a controlled study," Journal of Clinical and Experimental Dentistry, vol. 4, no. 5, pp. e286-e291, 2012.

[21] B. L. S. Picciani, B. Michalski-Santos, S. Carneiro et al., "Oral candidiasis in patients with psoriasis: correlation of oral examination and cytopathological evaluation with psoriasis disease severity and treatment," Journal of the American Academy of Dermatology, vol. 68, no. 6, pp. 986-991, 2013.

[22] M. Taheri Sarvtin, T. Shokohi, Z. Hajheydari, J. Yazdani, and M. T. Hedayati, "Evaluation of candidal colonization and specific humoral responses against Candida albicans in patients with psoriasis," International Journal of Dermatology, vol. 53, no. 12, pp. e555-e560, 2014.

[23] L. Chularojanamontri, C. Wongpraparut, P. Tuchinda et al., "Oral Candida colonization in Thai patients with psoriasis," Journal of the Medical Association of Thailand, vol. 99, no. 1, pp. 84-87, 2016.

[24] G. Javad, M. Taheri Sarvtin, M. T. Hedayati, Z. Hajheydari, J. Yazdani, and T. Shokohi, "Evaluation of Candida colonization and specific humoral responses against candida albicans in patients with atopic dermatitis," BioMed Research International, vol. 2015, Article ID 849206, 5 pages, 2015.

[25] D. Devore-Carter, S. Kar, V. Vellucci, V. Bhattacherjee, P. Domanski, and M. K. Hostetter, "Superantigen-like effects of a Candida albicans polypeptide," The Journal of Infectious Diseases, vol. 197, no. 7, pp. 981-989, 2008. 
[26] S. W. Kashem and D. H. Kaplan, "Skin immunity to Candida albicans," Trends in Immunology, vol. 37, no. 7, pp. 440-450, 2016.

[27] J. R. Naglik, J. P. Richardson, and D. L. Moyes, "Candida albicans pathogenicity and epithelial immunity," PLoS Pathogens, vol. 10, no. 8, article e1004257, 2014.

[28] R. A. Clark and C. Schlapbach, "TH9 cells in skin disorders," Seminars in Immunopathology, vol. 39, no. 1, pp. 47-54, 2017.

[29] M. Malakouti, G. E. Brown, E. Wang, J. Koo, and E. C. Levin, "The role of IL-17 in psoriasis," Journal of Dermatological Treatment, vol. 26, no. 1, pp. 41-44, 2015.

[30] A. W. Armstrong, M. Bukhalo, and A. Blauvelt, “A clinician's guide to the diagnosis and treatment of candidiasis in patients with psoriasis," American Journal of Clinical Dermatology, vol. 17, no. 4, pp. 329-336, 2016.

[31] D. M. Saunte, U. Mrowietz, L. Puig, and C. Zachariae, "Candida infections in patients with psoriasis and psoriatic arthritis treated with interleukin-17 inhibitors and their practical management," British Journal of Dermatology, vol. 177, no. 1, pp. 47-62, 2017.

[32] J. M. Rodríguez, K. Murphy, C. Stanton et al., "The composition of the gut microbiota throughout life, with an emphasis on early life," Microbial Ecology in Health and Disease, vol. 26, article 26050, 2015.

[33] C. A. O'Neill, G. Monteleone, J. T. McLaughlin, and R. Paus, "The gut-skin axis in health and disease: a paradigm with therapeutic implications," BioEssays, vol. 38, no. 11, pp. 1167-1176, 2016.

[34] C. L. Maynard, C. O. Elson, R. D. Hatton, and C. T. Weaver, "Reciprocal interactions of the intestinal microbiota and immune system," Nature, vol. 489, no. 7415, pp. 231-241, 2012.

[35] A. Pietrzak, I. Jastrzebska, G. Chodorowska et al., "Psoriasis vulgaris and digestive system disorders: is there a linkage?," Folia Histochemica et Cytobiologica, vol. 47, no. 3, pp. 517524, 2009.

[36] M. Chiu and C. Ni, "Psoriasis and comorbidities: links and risks," Clinical, Cosmetic and Investigational Dermatology, vol. 7, pp. 119-132, 2014.

[37] U. Mattsson, G. Warfvinge, and M. Jontell, "Oral psoriasis-a diagnostic dilemma: a report of two cases and a review of the literature," Oral Surgery, Oral Medicine, Oral Pathology and Oral Radiology, vol. 120, no. 4, pp. e183-e189, 2015.

[38] M. Fatahzadeh and R. A. Schwartz, "Oral psoriasis: an overlooked enigma," Dermatology, vol. 232, no. 3, pp. 319-325, 2016.

[39] M. Marcinkiewicz and S. Majewski, "The role of antimicrobial peptides in chronic inflammatory skin diseases," Advances in Dermatology and Allergology, vol. 1, no. 1, pp. 6-12, 2016.

[40] F. Niyonsaba, C. Kiatsurayanon, P. Chieosilapatham, and H. Ogawa, "Friends or foes? Host defense (antimicrobial) peptides and proteins in human skin diseases," Experimental Dermatology, vol. 26, no. 11, pp. 989-998, 2017.

[41] A. Abtin, L. Eckhart, R. Gläser, R. Gmeiner, M. Mildner, and E. Tschachler, "The antimicrobial heterodimer S100A8/S100A9 calprotectin is upregulated by bacterial flagellin in human epidermal keratinocytes," Journal of Investigative Dermatology, vol. 130, no. 10, pp. 24232430, 2010.
[42] C. F. Urban, D. Ermert, M. Schmid et al., "Neutrophil extracellular traps contain calprotectin a cytosolic protein complex involved in host defense against Candida albicans," PLoS Pathogens, vol. 5, no. 10, article e1000639, 2009.

[43] H. B. Schonthaler, J. Guinea-Viniegra, S. K. Wculek et al., "S100A8-S100A9 protein complex mediates psoriasis by regulating the expression of complement factor C3," Immunity, vol. 39, no. 6, pp. 1171-1181, 2013. 


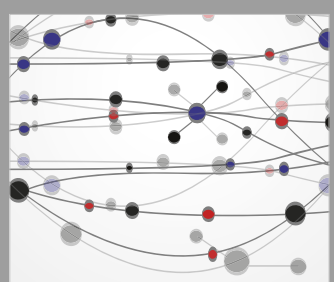

The Scientific World Journal
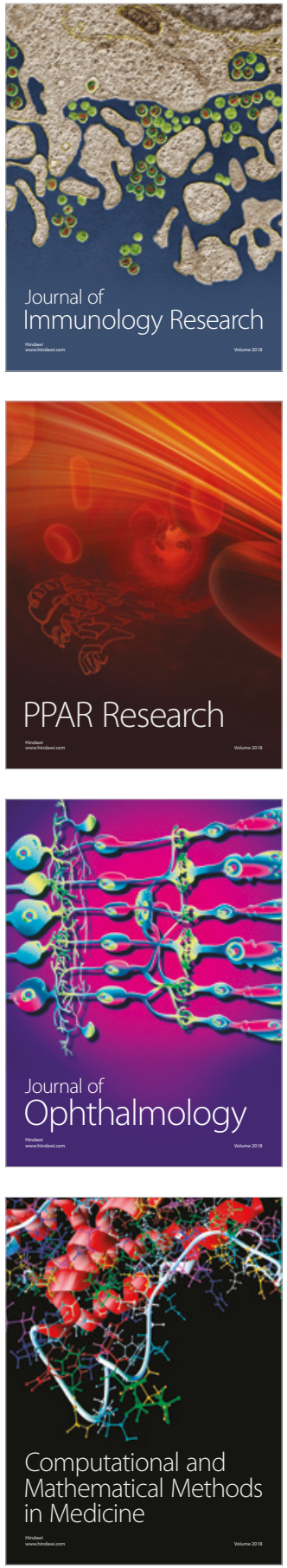

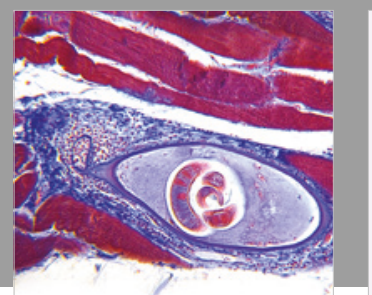

Gastroenterology Research and Practice

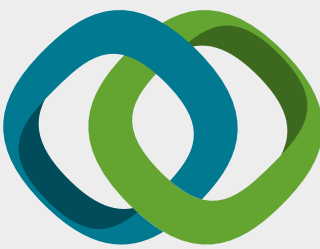

\section{Hindawi}

Submit your manuscripts at

www.hindawi.com
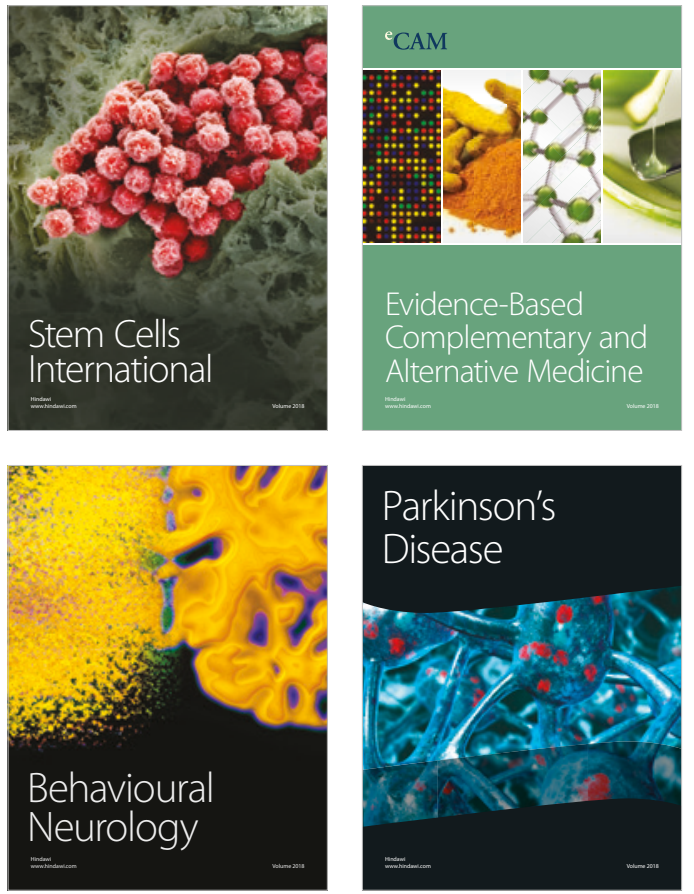

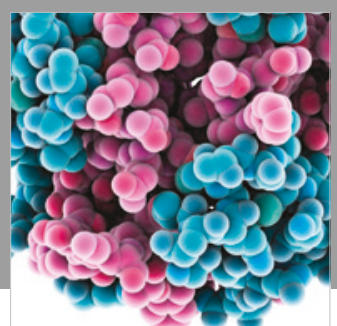

ournal of

Diabetes Research

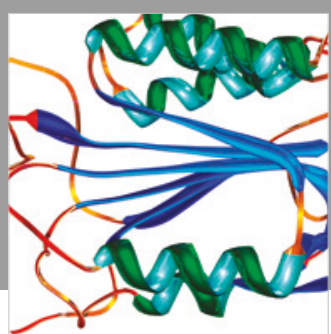

Disease Markers
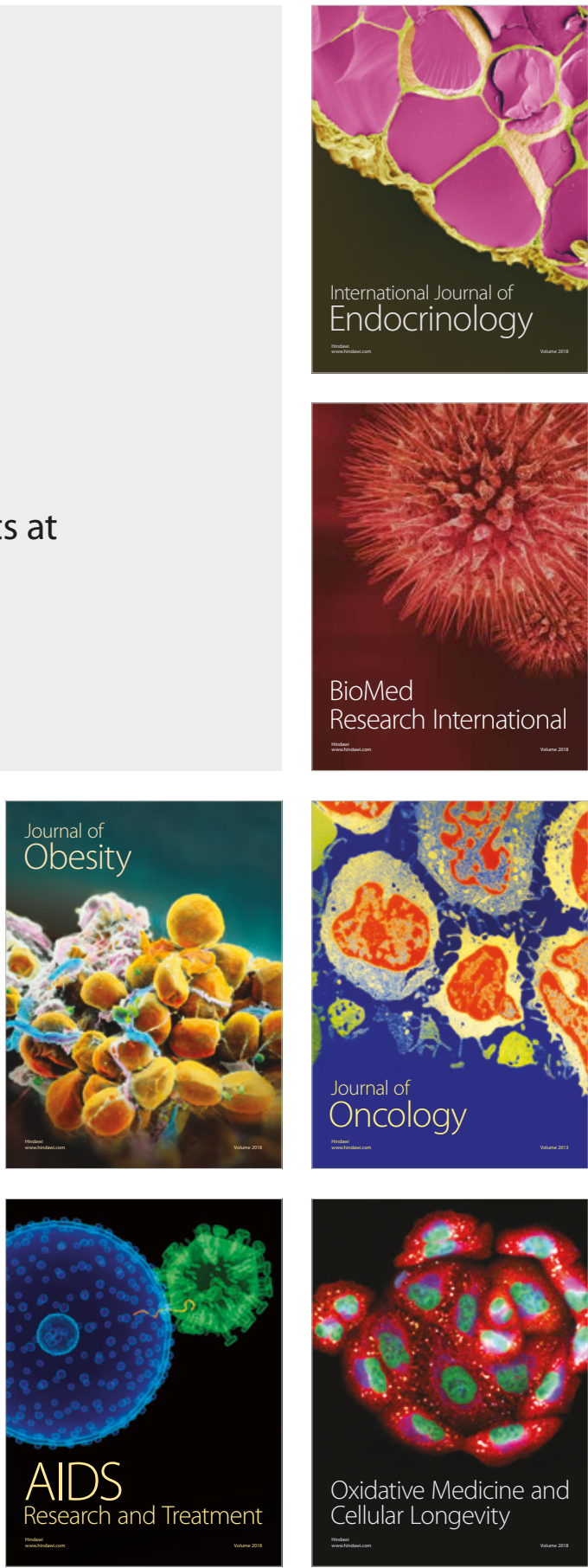